\title{
Research on Classification of Power Quality Disturbance Based on Weighted SVM Model
}

\author{
Xiangzheng $X \mathrm{u}^{1, \mathrm{a}}$, Jinwei $Y \mathrm{e}^{2, \mathrm{~b}}$ \\ ${ }^{1,2}$ Department of Electronics Engineering, East China Jiao-tong University, No.808 Shuanggang \\ Road, Nanchang, China \\ aemail:ecjtuxxz@163.com, bemail:1047960311@qq.com
}

Keywords: power quality, support vector machine, wavelet transform, fractal theory.

Abstract. In this paper, the mathematical models of the disturbance signal were to be designed. Adaptive LMS algorithm generalized morphological filter was set up based on the mathematical morphological, and the noise of power quality disturbance signal was managed. The different frequency energy distributions and fractal dimension of the power quality disturbance signals were extracted separately through the wavelet transform and the fractal theory. According to the time of the power quality disturbance to give different weights, to realize online training and testing. The simulation results show that structured the weighted support vector machine has very good classification effect for power quality disturbance.

\section{Introduction}

China's electric power system has developed into a multi-variable, multi-target and time varying system. In recent years, due to the power, the power system grid and load is undergoing profound changes, more and more nonlinear, impact and unbalanced load used in power system, such as arc furnace, electric railway, power electronics devices, leading to a series of grid power quality such as voltage fluctuations and flicker, harmonics, system frequency fluctuations and other disturbing problems. Therefore, the power quality problem has already caused the extensive concern. In this paper, according to the order of appearance of the disturbance signal settings from small to big weights, weighted support vector machine structure of power quality disturbance detection and recognition, improve recognition accuracy.

\section{Mathematical Model of Power Quality Disturbance Signal}

Voltage Sag. In Eq. 1, $0.1<\mathrm{a}_{1}<0.9$ is for sag amplitude, $\mathrm{T}<\mathrm{t}_{2}-\mathrm{t}_{1}<6 \mathrm{~T}$ is to sag duration, $\mathrm{t}_{1}$ is the start time occurs, $\mathrm{t}_{2}$ is the end time, $\mathrm{T}$ is the cycle [1].

$$
u(t)=\left\{\begin{array}{lc}
\sin \left(\omega_{0} t\right) & 0<t<t_{1} \text { or } t>t_{2} \\
a_{1} \sin \left(\omega_{0} t\right) & t_{1}<t<t_{2}
\end{array}\right.
$$

Voltage Swell. In Eq. 2, $1.1<\mathrm{a}_{1}<1.8$ is for swell amplitude, $\mathrm{T}<\mathrm{t}_{2}-\mathrm{t}_{1}<6 \mathrm{~T}$ is to swell duration, $\mathrm{t}_{1}$ is the start time of the occurrence, $t_{2}$ for the end time.

$$
u(t)=\left\{\begin{array}{lc}
\sin \left(\omega_{0} t\right) & 0<t<t_{1} \text { or } t>t_{2} \\
a_{1} \sin \left(\omega_{0} t\right) & t_{1}<t<t_{2}
\end{array}\right.
$$

Interruption. In Eq. 3, $0<\mathrm{a}_{1}<0.1$ is interrupted amplitude, $\mathrm{T}<\mathrm{t}_{2}-\mathrm{t}_{1}<6 \mathrm{~T}$ is for interruption duration, $\mathrm{t}_{1}$ is the start time occurs, $\mathrm{t}_{2}$ is the end time.

$$
u(t)=\left\{\begin{array}{lc}
\sin \left(\omega_{0} t\right) & 0<t<t_{1} \text { or } t>t_{2} \\
a_{1} \sin \left(\omega_{0} t\right) & t_{1}<t<t_{2}
\end{array}\right.
$$


Oscillatory Transients. In Eq. 4, a(0.1 0.8) is for the maximum amplitude oscillation, $\beta(0.1 \sim$ 0.5 ) is for the relative frequency fluctuation coefficient, $c$ is the attenuation coefficient of the oscillation, $\mathrm{T}<\mathrm{t}_{2}-\mathrm{t}_{1}<6 \mathrm{~T}$ is oscillation duration, $\mathrm{t}_{1}$ is the start time occurs, $\mathrm{t}_{2}$ is the end of the moment.

$$
u(t)=\sin \left(\omega_{0} t\right)+a e^{-c\left(t-t_{1}\right)} \sin \left(\beta \omega_{0} t\right)\left(u\left(t_{2}\right)-u\left(t_{1}\right)\right)
$$

Harmonics. In Eq. 5, $0.02<\mathrm{a}_{3}<1,0.02<\mathrm{a}_{5}<1,0.02<\mathrm{a}_{7}<1$ is amplitude of $3^{\text {rd }}, 5^{\text {th }}, 7^{\text {th }}$ harmonics, the harmonic content is generally to other relatively small, does not affect the analysis results.

$$
u(t)=\sin \left(\omega_{0} t\right)+a_{3} \sin \left(3 \omega_{0} t\right)+a_{5} \sin \left(5 \omega_{0} t\right)+a_{7} \sin \left(7 \omega_{0} t\right)
$$

Voltage Fluctuation. Which, Um is the amplitude of the carrier voltage power frequency, $V_{m} \cos \omega_{f} t$ is for sinusoidal voltage waveform, $\omega_{f}$ is for amplitude modulation wave voltage angular frequency, $\omega$ is the angular frequency of the voltage-frequency carrier [2].

$$
u(t)=\left(U_{m}+V_{m} \cos \omega_{f} t\right) \cos (\omega t)
$$

\section{Elimination Noise}

Assumed that the signal is $f_{0}$, the noise is $n$, the actual signals is $f=f_{0}+n$. If compared with the filtered signaly. Then, the error signal is

$$
e(n)=f_{0}-y \text {. }
$$

Minimize function is

$$
e(n)=E\left[e^{2}(n)\right]=E\left[\left(f_{0}-y\right)^{2}\right] .
$$

The estimate of a single square error gradient vector sequences instead of multiple sequences statistical average square error gradient [3].

$$
\frac{\partial E\left[e^{2}(n)\right]}{\partial \alpha}=\frac{\partial e^{2}(n)}{\partial \alpha}
$$

By steepest descent method available for the whole coefficient was iterative.

$$
\alpha_{i}(n+1)=\alpha_{i}(n)-\mu \frac{\partial E\left[e^{2}(n)\right]}{\partial \alpha_{i}}=\alpha_{i}(n)+2 \mu e(n) y_{i}(n)
$$

Where: $\mu$ is the convergence factor, the $\mu$ greater the value, the better convergence, convergence is faster. But the $\mu$ value is too large. The transition process will appear oscillation characteristics. When $\mu$ is equal to 0.03 , its weighting coefficient vector form of adaptive processing [3].

$$
A(n+1)=A(n)+2 \mu e(n) Y(n)
$$

Where, $A(n+1)=\left[\alpha_{1}(n+1), \alpha_{2}(n+1)\right], Y(n)=\left[y_{1}(n), y_{2}(n)\right]^{T}$

\section{Feature Extraction of Power Quality Disturbance Signal}

Extracting Energy Distribution Characteristics. Wavelet transform can be used to detect these strange signals, its multi-resolution signal decomposition of power quality disturbance signal can be decomposed into different frequency bands, and then time-domain analysis on the band. The energy 
distribution at different scales feature vector as a sample. Each energy decomposition scale is used by the following formula $[4,5]$.

$$
\begin{aligned}
& E_{c_{0}}=\sum_{j=1}^{N}\left|c_{o j}\right|^{2} \\
& E_{d_{0}}=\sum_{j=1}^{N}\left|d_{i j}\right|^{2} \quad i=1,2, \cdots, J
\end{aligned}
$$

Extract the Fractal Dimension. Fractal dimension as a quantitative characterization and basic parameters of the fractal is an important principle of the fractal theory. Because the box dimension does not reflect the unevenness of geometric objects, containing one or more points of the box has the same weight, the information dimension of this improvement in the above formula [5]. K-th hypercube probability $P_{k}(r)$ mind set of points $\Omega$ falling into the side length of $r$, r system accurate to state the amount of information needed to define the magnitude of entropy. The information dimension of the point set $(\Omega)$ is as follows.

$$
D_{I}=\lim _{r \rightarrow 0} \frac{I(r)}{\ln (1 / r)}
$$

\section{Weighted Support Vector Machine Online Training Algorithm}

Setting a training set $\left(x_{i}, y_{i}\right), x_{i} \in R^{n}, y_{i} \in\{-1,1\}, i=1,2, \cdots, 1$. Looking for a decision function $f(x)=\langle w \cdot x\rangle+b$ based on the training set. One side to make the gap between the two types of sample points is as large as possible, which is equivalent to $2 /\|w\|$ minimization. On the other hand so wrong points extent is as small as possible. So, the problem is transformed into solving the following convex quadratic programming problems [6,7].

$$
\begin{aligned}
& \min \frac{1}{2}\|w\|^{2}+c \sum_{1}^{l} \xi_{i} \\
& \text { st } \quad y_{i}\left(\left\langle w \cdot x_{i}\right\rangle+b \geq 1-\xi \quad \xi \geq 0, i=1,2, \cdots, l\right.
\end{aligned}
$$

Where: $\xi$ is the loss function, c is a given in advance to take the penalty parameter, which reflects the importance attached to the sample wrongly classified. In the standard support vector machine (C-SVM). The penalty coefficients of different samples are the same. However, in practical applications often found that certain large importance samples required for small training error. The importance of some of the samples is relatively low, allowable training error certain size. Given a weight $r_{i}$ for each training sample $x_{i}$ values according to the actual situation, each type of sample is assigned a weight $c_{y}$, the weighted support vector machine (W-SVM) can be described as follows [7].

$$
\begin{array}{ll} 
& \min \frac{1}{2}\|w\|^{2}+\sum_{1}^{l} c_{y i} r_{i} \xi_{i} \\
\text { st } \quad & y_{i}\left(\left\langle w \cdot x_{i}\right\rangle+b\right) \geq 1-\xi \quad \xi \geq 0, i=1,2, \cdots, l
\end{array}
$$

\section{Simulation Analysis of Power Quality Disturbance Recognition}

Power quality signal has generated 600 total set of samples, each power quality disturbances were 100 groups, which groups are used to train 40, the other 60 for detecting the group. In order to better simulate actual system disturbances power quality, adding signal to noise ration $15 \mathrm{db}$ voltage 
disturbances as Gaussian white noise. $\mathrm{Db}_{4}$ wavelet is chosen as the base Mallat wavelet decomposition. All signals are at the same sampling rate (256 points / cycle) sampling, sampling a total of 4096 points. Feature vectors were trained and tested based on two multi-class support vector machines, the test results are shown Table 1.

Table1 Support vector machine test results

\begin{tabular}{|c|c|c|c|}
\hline $\begin{array}{c}\text { Power Disturbance } \\
\text { Type }\end{array}$ & $\begin{array}{c}\text { The number of actual } \\
\text { disturbance }\end{array}$ & $\begin{array}{c}\text { The number of correct } \\
\text { classification }\end{array}$ & Correct rate (\%) \\
\hline Harmonics & 60 & 57 & 95 \\
\hline Voltage swell & 60 & 57 & 95 \\
\hline Voltage sag & 60 & 60 & 100 \\
\hline Voltage interruption & 60 & 60 & 98.33 \\
\hline Transient oscillations & 60 & 59 & 96.67 \\
\hline Voltage flicker & 60 & 58 & \\
\hline
\end{tabular}

As can be seen from the table, the online M-SVM can well identify different types of power disturbances. Recognition accuracy rate is more than $95 \%$, which shows the effectiveness of the proposed method.

\section{Conclusions}

This paper based on generalized morphological filter of adaptive LMS algorithm, power quality disturbance signal denoising can be improved. Power quality disturbance signals were processed to extract the energy distribution of the disturbance signal based on wavelet transform with multiresolution. Meanwhile, based on fractal geometry theory, the power quality disturbances is extracted local variance dimension. Combine the two together constitute the power quality disturbance signal feature vectors. According to the time of power quality disturbances occurring different weights were given, to realize online training. Studies have shown that the weighted support vector machine has very good classification accuracy for power quality disturbance.

\section{Acknowledgements}

This work was supported by the project "Research of power quality disturbance recognition based on on-line training of weighted SVM". The project is No. GJJ13353. The authors are grateful to the Jiangxi Province Education Department of China for their help.

\section{References}

[1] H.L. Wang, in: Research on Dynamic Power Quality Disturbance Recognition Based on Wavelet Entropy and SVM, North China Electric Power University (2007).

[2] X.D. Yu, L.A. Zhou: Power System Protec. and Con. Vol.38(2010),p.15

[3] J. Shi, C. Huang, Y. Li: Proceeding s of the CSU-EPSA. Vol.20(2008),p.86

[4] Y.P. Gong, Y.W. Liu, L.Y. Wu: Power System Tech. Vol.35 (2011), p.135

[5] W.B. Hu, K.C. Li: Relay. Vol.36(2008), p. 45

[6] Y.P. Qin, Q. Ai, W.J. Liu: Research of Comput. Vol.27(2007), p.32

[7] Y.T. Xu, Z.Q. Qi, L.S. Wang: Journal of Yantai University (Natural Science and Engineering Edition), Vol.24(2011), p.65 